\title{
Analytical estimation of flow-induced forces in endovascular grafts and design methodology for a tissue-engineered endovascular attachment mechanism
}

\author{
E.A. Finol ${ }^{1,2}$, K.G. Marra ${ }^{1,2,3}$ \& C.H. Amon ${ }^{1,2,4}$ \\ 'Institute for Complex Engineered Systems \\ ${ }^{2}$ Biomedical Engineering \\ ${ }^{3}$ Materials Science and Engineering \\ ${ }^{4}$ Mechanical Engineering, Carnegie Mellon University, U.S.A.
}

\begin{abstract}
Endovascular repair has emerged as an alternative, less-invasive surgical technique for the treatment of patients diagnosed with abdominal aortic aneurysms (AAAs). The anatomical pathway of blood flow in the abdominal aorta is restored by the implantation of an endovascular graft (EVG), depressurizing the aneurysm and initiating a remodeling process of the diseased aortic tissue. The short-term results of endovascular grafting are promising, but its long-term success has been compromised by the occurrence of graft migration or detachment, which induce endoleaks or incomplete occlusion of the aneurysm from the blood circulation. The forces induced by the blood as it flows through the graft are believed to be a factor of probable cause in the migration of the graft downstream and the partial detachment of its proximal and distal anchoring points. The purpose of this study is to utilize analytical tools to provide an estimation of the forces required to secure the graft proximally when relying on flow-induced stresses alone, and to describe the design methodology of a tissueengineered proximal attachment mechanism that is capable of withstanding these forces. Composites of synthetic and native biodegradable polymers are examined as biomaterials for the attachment mechanism of the graft.
\end{abstract}




\section{2}

Simulations in Biomedicine $V$

\section{Introduction}

The first successful AAA resection and homograft replacement dates back to 1951 [1], then considered a promising less-invasive method of treatment. What is now recognized as endovascular grafting was first reported by Parodi et al. [2] as a procedure that avoids the extensive tissue dissection associated with traditional open repair. Even though the initial and short term results for endovascular repair of AAAs are promising, there are challenges to overcome for its use for the longterm treatments. One of the common problems encountered is the incidence of incomplete aneurysm exclusion, also known as an endoleak, which originates from the sites of either proximal or distal fixation. Type I endoleaks are the result of failure to exclude the aneurysm from central circulation [3], which may lead to acute aortic occlusion or perigraft flow and to repressurization of the aneurysm sac that can eventually cause rupture of the AAA [4]. This type of endoleak is consistently associated with the failure of the attachment mechanism of the graft to the inner wall of the aorta and to the in-vivo downstream migration of the graft. However, the forces that cause endovascular grafts to migrate in-vivo are unknown [5].

All known commercial grafts utilize metallic components based on either a passive or an active attachment mechanism. Passive attachments allow the graft to be held in place solely by the frictional force provided by the graft's metallic frame. Active attachments consist of metallic hooks at the proximal and distal ends that hold the graft in place by inserting themselves into the artery wall. One of the factors believed to cause failure of the attachment mechanism is the fatigue of the metallic structure [6], which leads to detachment of the hooks in active mechanisms and to detachment of the metallic structure from the graft fabric in passive mechanisms. Blood flow patterns and the forces induced by blood flow through the aorta are hypothesized to be one of the probable factors involved in the appearance of endoleaks. In a recent workshop on pre-clinical testing for endovascular grafts [6], the anatomical pathway for blood flow through an AAA was considered as a characteristic that could affect function or performance of an endovascular graft. Moreover, the presence of an implanted graft is predicted to alter pre-implant flow patterns, which may have an influence on potential endoleaks.

To address the recurrent problem of Type I endoleaks in endovascular grafts used for AAA repair, we propose a tissue engineering approach that will provide stability and durability of the proximal attachment site. The objective of this investigation is to develop a tissue-engineered attachment mechanism for endovascular grafting applications. Analytical techniques are utilized in this work to estimate the reaction forces required to secure an endovascular graft post-implantation. Experimental techniques used to determine the adhesion properties of the tissue-engineered material to the attachment mechanism are also described. This work focuses on the (i) analytical evaluation of the flow-induced forces the attachment site of an endovascular graft experiences after its implantation for AAA repair, and (ii) design approach for a biocompatible material that will allow its integration with either passive or active EVG 
attachment systems, while promoting tissue ingrowth and intralumenal anchorage with the aortic wall.

\section{Methodology}

Following is an outline of the methods followed to (i) evaluate the types and magnitudes of flow-induced forces the attachment site of an endovascular graft experiences after its implantation for AAA repair, and (ii) describe the design approach necessary to develop a proximal attachment mechanism that will allow its integration with either passive or active attachment systems, while promoting tissue ingrowth and intralumenal anchorage with the aortic wall.

\subsection{Analytical modeling}

Analytical graft models, based on the integral relations of fluid particles applied to control volumes, are used in this investigation to provide an initial estimate of the reaction forces that attempt to dislodge the graft from its proximal attachment site. These forces are calculated on the basis of a two-dimensional force system and obtained at peak flow during the cardiac cycle, which corresponds to the maximum blood flow rate. The analytical models have allowed us to perform a parametric study of the variation of fluid forces with inlet and outlet graft diameters, angulation of the graft limbs, and the change in physiological peak blood flow rates.

The control volume geometries are constructed to investigate the forces acting on an endovascular graft using a steady-state peak flow design of a bifurcated stent-graft with dimensions similar to typical AneuRx ${ }^{\circledR}$ and Ancure ${ }^{\circledR}$ grafts. A balance of fluid forces is performed in each control volume to obtain the resultant reaction forces on the graft in a post-implantation setting. A schematic of a representative control volume and the forces induced by the flow are shown in Figure 1. The control volume is modeled as stationary and the blood flow properties constant and uniform. Blood is assumed to be incompressible and Newtonian, which is a reasonable modeling assumption for large arteries. The inlet velocity $\mathrm{V}_{1}$ in the laminar regime is taken as the peak flow velocity for average resting hemodynamic conditions $[7,8]$. In the turbulent regime, the inlet velocity is taken as $V_{1}=0.6 \mathrm{~m} / \mathrm{s}$, a value within the range of observed peak systolic velocities under average exercise conditions. An inlet pressure $P_{1}=120$ $\mathrm{mmHg}(16 \mathrm{kPa})$ is assumed for all the analyses.

The application of Reynolds' Transport Theorem [9] dictates the analytical balance of forces. Mass conservation over the control volume is given by Eq. (1):

$$
0=\frac{\mathrm{d}}{\mathrm{dt}}\left(\int_{\mathrm{cv}} \rho \mathrm{d} \forall\right)+\int_{\mathrm{cs}} \rho\left(\overrightarrow{\mathrm{V}}_{\mathrm{r}} \cdot \hat{\mathrm{n}}\right) \mathrm{dA}
$$

which is simplified as

$$
V_{2}=\beta\left(\frac{D_{1}}{D_{2}}\right)^{2} V_{1} ; \quad V_{3}=(1-\beta)\left(\frac{D_{1}}{D_{3}}\right)^{2} V_{1}
$$




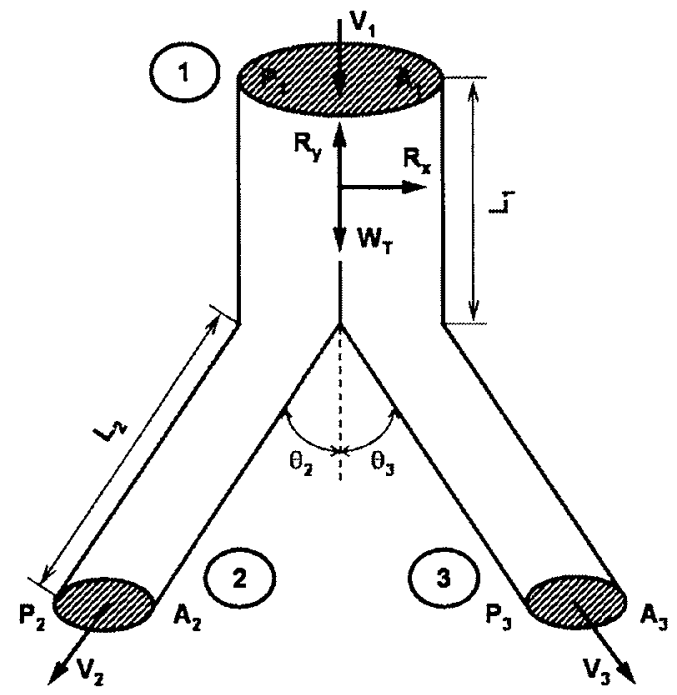

Figure 1: Control volume of an axisymmetric bifurcated endovascular graft illustrating the flow-induced forces and geometric parameters.

for which $\beta=0.5$, resulting in $V_{2}=V_{3}$, and $\sin \theta_{2}=-\sin \theta_{3}$ and $\cos \theta_{2}=\cos \theta_{3}$. The latter is representative of an axisymmetric graft, the only type of graft geometry considered in this study.

Energy conservation is performed over the control volume as indicated by Eq. (3) in its most general form:

$$
\begin{aligned}
\dot{\mathrm{Q}}-\dot{\mathrm{W}}_{\mathrm{s}}-\dot{\mathrm{W}}_{\mathrm{v}}= & \frac{\mathrm{d}}{\mathrm{dt}}\left[\int_{\mathrm{cv}}\left(\hat{\mathrm{u}}+\frac{1}{2} \mathrm{~V}^{2}+\mathrm{gz}\right) \rho \mathrm{d} \forall\right] \\
& +\int_{\mathrm{cs}}\left(\hat{\mathrm{h}}+\frac{1}{2} \mathrm{~V}^{2}+\mathrm{gz}\right) \rho(\hat{\mathrm{V}} \cdot \hat{\mathrm{n}}) \mathrm{dA}
\end{aligned}
$$

This is simplified for steady flow as follows:

$$
\frac{\mathrm{P}_{1}}{\gamma}+\alpha_{1} \frac{\mathrm{V}_{1}^{2}}{2 \mathrm{~g}}+\mathrm{y}_{1}=\left(\frac{\mathrm{P}_{\mathrm{i}}}{\gamma}+\alpha_{\mathrm{i}} \frac{\mathrm{V}_{\mathrm{i}}^{2}}{2 \mathrm{~g}}+\mathrm{y}_{\mathrm{i}}\right)+\mathrm{f} \frac{\mathrm{L}_{1, \mathrm{i}}}{\mathrm{D}_{\mathrm{i}}} \frac{\mathrm{V}_{\mathrm{i}}^{2}}{2 \mathrm{~g}}
$$

where $\mathrm{i}=2,3$ and $\mathrm{L}_{1, \mathrm{i}}=\mathrm{L}_{1}+\mathrm{L}_{\mathrm{i}}$. Also, $\mathrm{D}_{2}=\mathrm{D}_{3}, \alpha_{2}=\alpha_{3}$ and $\mathrm{y}_{2}=\mathrm{y}_{3}$ due to the configuration and symmetry of the graft, resulting in $\mathrm{P}_{2}=\mathrm{P}_{3}$. The inclusion of kinetic correction factors $\left(\alpha_{i}\right)$ and the appropriate calculation of the friction factor (f) are improvements with respect to the control-volume analyses reported by Liffman et al. [10]. Kinetic correction factors are defined by Eq. (5):

$$
\alpha=\frac{1}{\mathrm{~A}} \int\left(\frac{\mathrm{u}}{\mathrm{V}}\right)^{3} \mathrm{dA}
$$

where $\mathrm{u}$ is the instantaneous axial velocity and $\mathrm{V}$ is the spatially-averaged velocity at a cross-section. For laminar parabolic flow, $\alpha=2.0$. For turbulent 
flow, $\alpha$ generally varies in the range $1.04<\alpha<1.11$. For the purpose of this study it is assumed that $\alpha_{\text {turbulent }}=1.075$.

The friction factor is given by Eq. (6):

$$
\mathrm{f}=\frac{8 \tau_{\mathrm{w}}}{\rho \mathrm{V}^{2}}
$$

where $\tau_{w}$ is the flow-induced wall shear stress and $\rho$ is the density of blood. Since wall shear stress varies linearly in fully-developed pipe flow conditions in the laminar regime, Eq. (6) is simplified as $f=64 / \mathrm{Re}$ for laminar flow, where $\operatorname{Re}=\rho V_{1} D_{1} / \mu$ is the Reynolds number. For turbulent flow, we have used the Blasius correlation [9], which states that $\mathrm{f}=0.316 \mathrm{Re}^{-1 / 4}$ for $400<\operatorname{Re}<10^{5}$.

Conservation of linear momentum is also performed over the control volume as indicated by the general integral balance of forces in Eq. (7):

$$
\frac{\mathrm{d}}{\mathrm{dt}}(\mathrm{mV})_{\text {syst }}=\sum \overrightarrow{\mathrm{F}}=\frac{\mathrm{d}}{\mathrm{dt}}\left(\int_{c \mathrm{v}} \overrightarrow{\mathrm{V}} \rho \mathrm{d} \forall\right)+\int_{\mathrm{cs}} \overrightarrow{\mathrm{V}} \rho\left(\overrightarrow{\mathrm{V}}_{\mathrm{r}} \cdot \hat{\mathrm{n}}\right) \mathrm{dA}
$$

This results in the following expressions for the forces in the $\mathrm{x}$ and $\mathrm{y}$ directions:

$$
\begin{aligned}
\sum F_{x}= & R_{x}+P_{2} A_{2} \sin \theta_{2}+P_{3} A_{3} \sin \theta_{3}=-\rho V_{2}^{2} A_{2} \sin \theta_{2}-\rho V_{3}^{2} A_{3} \sin \theta_{3} \\
\sum F_{y}= & R_{y}+P_{2} A_{2} \cos \theta_{2}+P_{3} A_{3} \cos \theta_{3}-P_{1} A_{1} \\
& -W_{T}=\rho V_{1}^{2} A_{1}-\rho V_{2}^{2} A_{2} \cos \theta_{2}-\rho V_{3}^{2} A_{3} \cos \theta_{3}
\end{aligned}
$$

where $\mathrm{W}_{\mathrm{T}}$ is the total weight of the fluid and the graft, given by $\mathrm{W}_{\mathrm{T}}=\mathrm{W}_{\mathrm{f}}+\mathrm{W}_{\mathrm{G}}$ $=\rho g \forall_{\mathrm{G}}+\mathrm{m}_{\mathrm{G}} \mathrm{g}, \forall_{\mathrm{G}}$ being the internal volume of the graft (lumen for the flow).

Substituting Eqs. (2) and (4) into Eq. (8) yields the following expression for the horizontal and vertical reaction forces $R_{x}$ and $R_{y}$ :

$$
\begin{aligned}
& \mathrm{R}_{\mathrm{x}}=0 \\
& \mathrm{R}_{\mathrm{y}}=\left(\mathrm{P}_{1}+\rho \mathrm{V}_{1}^{2}\right) \frac{\pi}{4} \mathrm{D}_{1}^{2}+\mathrm{W}_{\mathrm{T}}-\left[\mathrm{P}_{2}+\frac{\rho}{4}\left(\frac{\mathrm{D}_{1}}{\mathrm{D}_{2}}\right)^{4} \mathrm{~V}_{1}^{2}\right] \frac{\pi}{2} \mathrm{D}_{2}^{2} \cos \theta_{2}
\end{aligned}
$$

where $P_{2}=P_{1}+\rho \frac{V_{1}^{2}}{2}\left[\alpha_{1}-\frac{1}{4}\left(\frac{D_{1}}{D_{2}}\right)^{4}\left(\alpha_{2}+f \frac{L_{1,2}}{D_{2}}\right)\right]+\gamma\left(y_{1}-y_{2}\right)$.

Average blood properties are taken as $\rho=1,050 \mathrm{~kg} / \mathrm{m}^{3}$ and $\mu=0.00319 \mathrm{~Pa} \mathrm{~s}$. The geometry and properties of the graft are: $\mathrm{L}_{1}=7.75 \mathrm{~cm}, \mathrm{~L}_{2}=\mathrm{L}_{3}=12.25 \mathrm{~cm}$, $\mathrm{m}_{\mathrm{G}}=0.00785 \mathrm{Kg}[10]$. For the parametric analyses, the inlet diameter varies in the range $2.0 \mathrm{~cm}<\mathrm{D}_{1}<3.0 \mathrm{~cm}$. The outlet diameters vary in the range $1 / 2 D_{1}<D_{2}=D_{3}<1 / 6 D_{1}$ and the angulation of the axisymmetric limbs varies in as $0^{\circ}<\theta_{2}=\theta_{3}<45^{\circ}$. The peak Reynolds for the pulsatile curve in Maier et al. [7] is fixed, from which $V_{1}$ is calculated for the laminar flow studies.

\subsection{Tissue-engineering approach}

Our approach is to engineer a material that will cover the stent or hook, and in application, have tight apposition with the aortic wall upon graft deployment. Stents are typically made of metals or shape-based metal alloys [11]. 
Modifications to the metals include covering the stents with synthetic polymers such as Dacron or expanded poly(tetrafluoroethylene) [12, 13]. Biodegradable polymers have also been examined as stents $[14,15]$. Recently, porcine small intestinal submucosa (SIS) has been used as a stent coating to treat AAA [12, 16]. SIS is obtained from the middle layer of the small intestine, and is an acellular, collagen-based biomaterial that becomes remodeled with the tissue. By attaching a tissue-engineered scaffold at the ends of the EVG, the graft can perform its attachment function in the short term, while allowing the engineered biomaterial to promote ingrowth from the aortic wall for long-term anchorage.

\subsection{Experimental verification}

EVGs will be impregnated with poly(vinyl alcohol), (PVA), a biocompatible hydrogel, and SIS will be incorporated into the hydrogel. Recently, Oka has reported the successful results of impregnating titanium meshes with poly(vinyl alcohol) hydrogels for cartilage replacement [17]. We will utilize SIS to enable the outer surface of the EVG to remodel within the aortic wall, while the PVA hydrogel will interlock the SIS to the EVG. SIS has shown promising results as an artificial vascular graft [18]. The use of a hydrogel is necessary as recent studies demonstrated poor attachment of SIS to AAA stents [12].

\section{Results and discussion}

The axysimmetric geometry of the grafts modeled in the present investigation has an effect on the change in momentum of the flow caused by the inlet boundary conditions and the tapered body of the graft, which results in a zero horizontal restraint force, as yielded by Eq. (9a). The force required to maintain the graft in place due to blood flowing at a steady peak systolic rate is vertical, oriented in the streamwise direction and is highly dependent on the inlet pressure and velocity.

From the parametric studies conducted, we have chosen a representative configuration [10] for which $\mathrm{D}_{1}=3.0 \mathrm{~cm}, \mathrm{D}_{2,3}=1.0 \mathrm{~cm}$ and $\theta_{2,3}=15^{\circ}$. The analysis is based on laminar and turbulent flows, with the patient standing or lying horizontally (absence of gravitational forces). The results are:

a) Laminar regime for which $V_{1}=0.26 \mathrm{~m} / \mathrm{s}$ at peak systolic flow, $P_{1}=16 \mathrm{kPa}$, resulting in $\mathrm{V}_{2,3}=1.17 \mathrm{~m} / \mathrm{s}$.

- Patient standing, $\mathrm{R}_{\mathrm{y}}=9.51 \mathrm{~N}$

- Patient lying horizontally, $\mathrm{R}_{\mathrm{y}}=8.97 \mathrm{~N}$

b) Turbulent regime for which $\mathrm{V}_{1}=0.60 \mathrm{~m} / \mathrm{s}$ at peak systolic flow, $P_{1}=16 \mathrm{kPa}$, resulting in $\mathrm{V}_{2,3}=2.70 \mathrm{~m} / \mathrm{s}$.

- Patient standing, $\mathrm{R}_{\mathrm{y}}=9.53 \mathrm{~N}$

- Patient lying horizontally, $\mathrm{R}_{\mathrm{y}}=9.00 \mathrm{~N}$

This demonstrates the negligible increase in restraint force when considering turbulent flow in control-volume graft studies. A complete analysis for laminar flow with the inlet diameter $D_{1}=3.0 \mathrm{~cm}$ is shown in Figure 2 . The results show 
an increase in vertical restraint force with hypothetical inlet-outlet diameter ratios that may not be available in all commercial endovascular grafts. A typical Ancure ${ }^{\circledR}$ graft measures $D_{1}=2.6 \mathrm{~cm}$ and $D_{2,3}=1.3 \mathrm{~cm}$, while a typical AneuRx ${ }^{\circledR}$ graft measures $\mathrm{D}_{1}=2.8 \mathrm{~cm}$ and $\mathrm{D}_{2,3}=1.6 \mathrm{~cm}$. A comparison of restraint force variation for these grafts is shown in Figure 3 for laminar and turbulent flows.

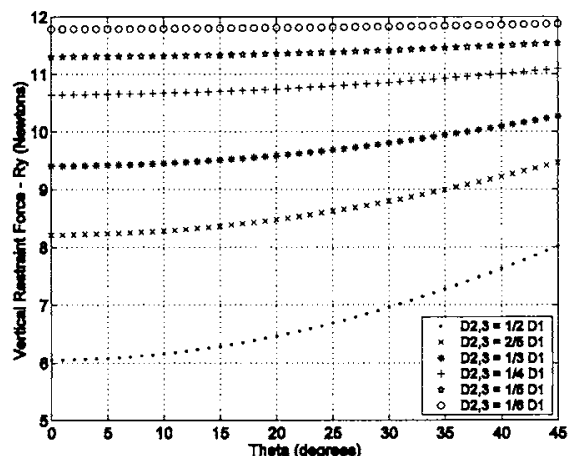

(a)

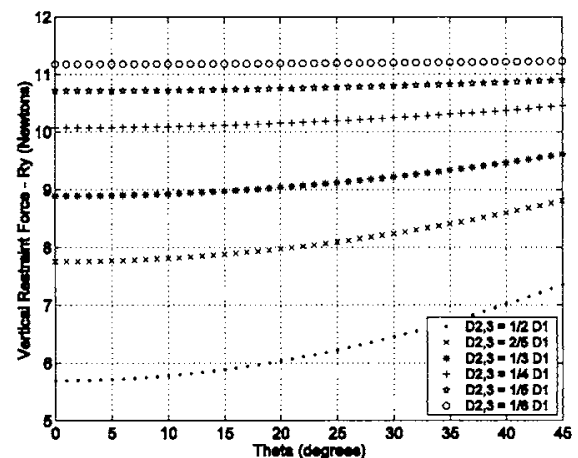

(b)

Figure 2: Variation of vertical restraint force $R_{y}$ for laminar flow and $D_{1}=3.0$ $\mathrm{cm}$ for a patient (a) standing and (b) lying horizontally.

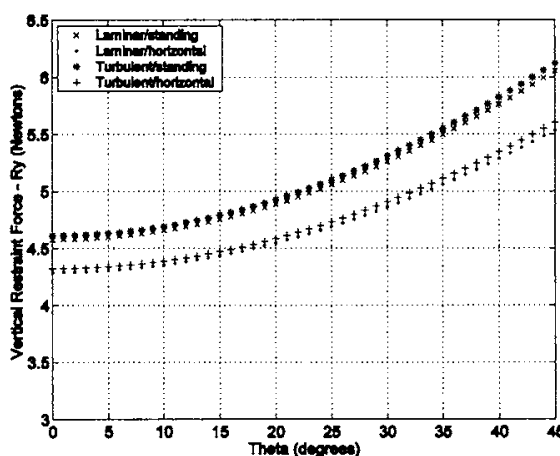

(a)

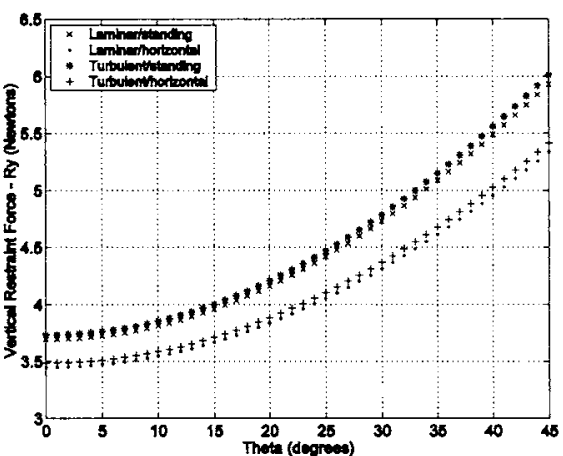

(b)

Figure 3: Variation of vertical restraint force $R_{y}$ for (a) $D_{1}=2.6 \mathrm{~cm}$ and $\mathrm{D}_{2,3}=1.3 \mathrm{~cm}$, and (b) $\mathrm{D}_{1}=2.8 \mathrm{~cm}$ and $\mathrm{D}_{2,3}=1.6 \mathrm{~cm}$.

Experimental measurements of forces required to displace the proximal end of a graft are found in the literature. Lawrence-Brown et al. [19] have determined that the force required to dislodge a graft from its proximal end when relying on an unbarbed stent is $5-6 \mathrm{~N}$. The displacement force to extract anchored grafts in cadaveric aortas is $2-3.4 \mathrm{~N}$ for a smooth stent, $7.4-10.8 \mathrm{~N}$ for a stent with four weak hooks and barbs, and $17.1-27.9 \mathrm{~N}$ for a stent with eight strengthened hooks and barbs [5]. Morris et al. [20] report a force of $2.5 \mathrm{~N}$ as that required to cause stent-graft migration in silicon aortic aneurysms when relying on up to 


\section{8}

Simulations in Biomedicine $V$

three smooth stents, one at each end of the graft. Based on these findings and a control-volume analysis of peak systolic flow rate, blood flow alone may cause stent-graft migration or detachment if relying on a passive attachment mechanism or on an active attachment mechanism based on any combination of up to four hooks and barbs at the proximal end.

SIS has been harvested following a procedure by Hadlock et al. [21] Briefly, entire adult rat small intestines were removed and washed with saline. The intestines were cut longitudinally and the intralumenal mucosa was exposed. A scalpel was used to mechanically remove the mucosa. The strip was turned over and similarly, a scalpel was used to gently remove the serosa. The remaining submucosa was sterilized in $0.1 \%$ peracetic acid, and stored in PBS at $4^{\circ} \mathrm{C}$ until use. Figure 4(a) is a digital image of the isolated rat SIS and Figure 4(b) is a scanning electron micrograph of the rat SIS.

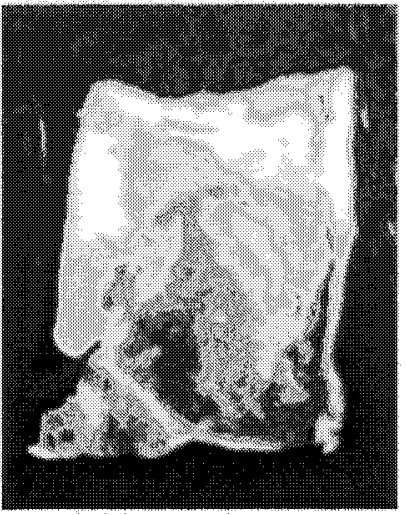

(a)

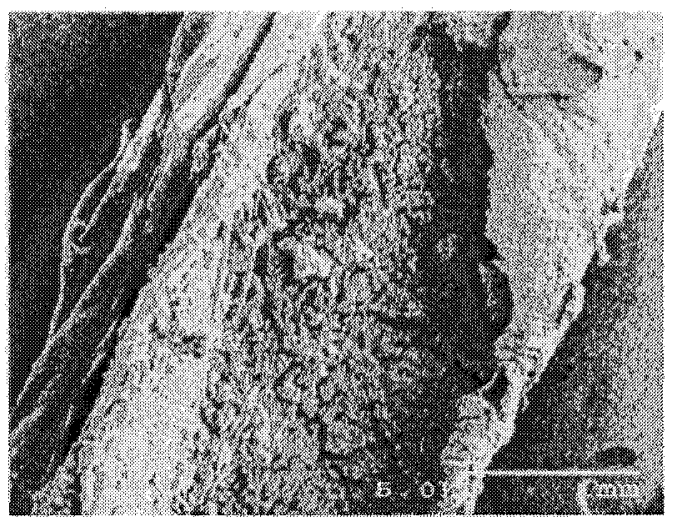

(b)

Figure 4: Rat small intestinal submucosa (SIS): (a) digital image and (b) scanning electron micrograph.

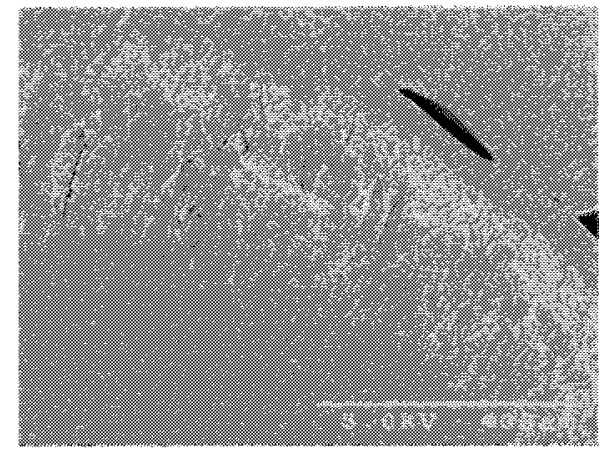

(a)

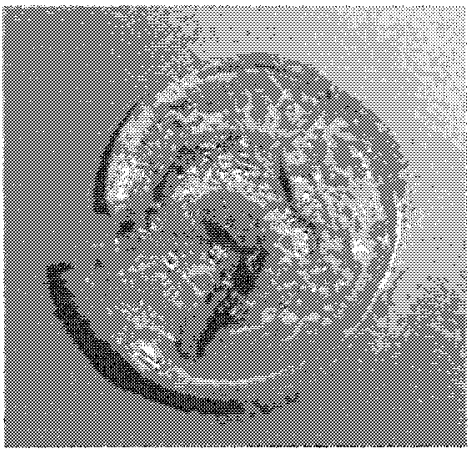

(b)

Figure 5: SIS incorporated within various polymer matrices: (a) PVA and (b) cross-linked PVA hydrogel. 
Incorporation of SIS into PVA has been achieved using a "sandwich" technique. PVA was dissolved in water and poured into a circular Teflon mold. After a specified time period, a sheet of SIS was applied to the surface of the PVA. An additional layer of aqueous PVA was added, and the "sandwich" was cross-linked with glutaraldehyde. A scanning electron micrograph of the PVA/SIS composite is shown in Figure 5(a), and a digital image is shown if Figure 5(b). Additional experiments include the incorporation of SIS within an FDA-approved biodegradable polyester (e.g., polycaprolactone), as well as utilizing non-toxic cross-linking agents and examining the adhesion properties between the composite layers.

\section{Conclusions}

The long-term efficacy of endovascular repair (EVAR) of abdominal aortic aneurysms (AAAs) is under examination. Endoleaks type I remain problematic and can result in rupture. Our approach seeks to improve upon EVAR through a powerful combination of analytical modeling of flow-induced forces and tissueengineering design of a biocompatible attachment mechanism.

Based on a two-dimensional force system of control-volume integral relations, it was determined that the restraint force exerted on an axisymmetric endovascular graft due to peak systolic blood flow alone is a streamwise force that may detach the graft and cause migration downstream depending on the type of traditional attachment mechanism utilized. Such force is due to the inlet pressure and velocity conditions, the change in momentum of the fluid due to the ratio of inlet to outlet cross-sectional areas, and the angulation of the limbs of the graft. The analyses presented in this investigation are an initial approximation for a subsequent study of the complex flow-induced stress field that can be accurately estimated by means of computational fluid dynamics modeling. The present work does not take into account asymmetry of the endovascular graft, three-dimensional force systems, flow pulsatility, mechanical interaction with the aneurysm, hypertension, and the presence of other types of endoleaks. The forces induced by the flow are general guidelines for our design of a tissue-engineered attachment mechanism that can prevent the appearance of endoleaks type I.

To the best of our knowledge, no existing graft manufacturer utilizes a tissue engineering approach for the development of proximal or distal attachment mechanisms. Commercial grafts attempt to address the appearance of endoleaks by providing a variety of metallic components in combination with modular graft bodies manufactured with different fabrics. The active attachment-based grafts, for example, consist of six to eight hooks that provide radial support proximally and either barbs and/or stents for additional stability of the mechanism. Placing additional hooks and barbs on a stent increases the volume of the attachment mechanism and makes its delivery more difficult during surgery. The advantage of using a tissue-engineered material in combination with an existing attachment mechanism is the provision of a long-term solution based on the induced natural growth of the aortic tissue surrounding the material, by the time that the active metallic components or the passive metallic grids would fail due to fatigue. 


\section{Acknowledgments}

The authors gratefully acknowledge the Pittsburgh Tissue Engineering Initiative, Inc. (KGM). Funding for this work is provided in part by the Pennsylvania Infrastructure Technology Alliance from the Commonwealth of Pennsylvania's Department of Community and Economic Development (EAF). AneuRx® and Ancure ${ }^{\circledR}$ are registered trademarks of Medtronic Corp. and Guidant Corp., respectively.

\section{References}

[1] Dubost, C., Allary, M., and Oeconomos, N. Resection of an Aneurysm of the Abdominal Aorta: Reestablishment of the continuity by a preserved human arterial graft, with result after five months. Archives of Surgery, 64, pp. 405-408, 1952.

[2] Parodi, J., Palmaz, J., and Baroni, H. Transfemoral intraluminal graft implantation of abdominal aortic aneurysms. Annals of Vascular Surgery, 5, pp. 491-499, 1991.

[3] Ohki, T., Veith, F., Marin, M., Sanchez, L., Suggs, W., Cynamon, J., and Wain, R. Transluminally placed endovascular grafts for the treatment of aortoiliac aneurysms. Diagnosis and Treatment of Aortic and Peripheral Arterial Aneurysms, ed. by Calligaro, K., Dougherty, M., and Hollier, L., W.B. Saunders Company, Ltd., Philadelphia, PA, pp. 113-131, 1999.

[4] Harris, P.L., Vallabhaneni, S.R., Desgranges, P., Becquemin, J.P., van Marrewijk, C., and Laheij, R.J. Incidence and risk factors of late rupture, conversion, and death after endovascular repair of infrarenal aortic aneurysms: the EUROSTAR experience. Journal of Vascular Surgery, 32(4), pp. 739-749, 2000.

[5] Malina, M., Lindblad, B., Ivancev, K., Lindh, M., Malina, J., and Brunkwall, J. Endovascular AAA exclusion: will stents with hooks and barbs prevent stent-graft migration? Journal of Endovasular Surgery, 5, pp. 310-317, 1998.

[6] Department of Health and Human Services. Workshop on Pre-Clinical Testing for Endovascular Grafts. Office of Device Evaluation, Food and Drug Administration, Rockville, MD, 2001.

[7] Maier, S., Meier, D., Boesiger, P., Moser U., and Vieli, A. Human abdominal aorta: comparative measurements of blood flow with MR imaging and multigated Doppler US. Radiology, 171, pp. 487-492, 1989.

[8] Pedersen, E., Sung, H., Burlson, A., and Yoganathan, A. Two-dimensional velocity measurements in a pulsatile flow model of the normal abdominal aorta simulating different hemodynamic conditions. Journal of Biomechanics, 26, pp. 1237-1247, 1993.

[9] White, F. Fluid Mechanics, Third Edition, McGraw-Hill, Inc., New York, NY, pp. 110-166, 1994. 
[10] Liffman, K., Lawrence-Brown, M., Semmens, J., Bui, A., Rudman, M., and Hartley, D. Analytical modeling and numerical simulation of forces in an endoluminal graft. Journal of Endovascular Therapy, 8, pp. 358-371, 2001.

[11] Pescatore, P., Meier-Willersen, H., and Manegold, B. A severe complication of the new self-expanding spiral nitinol biliary stent. Endoscopy, 29, pp. 413-415, 1997.

[12] Yamada, K., Pavcnik, D., Uchida, B.T., Timmermans, H.A., Corless, C.L., Yin, Q.W., Yamakado, K., Park, J.W., Rösch, J., Keller, F.S., Sato, M., and Yamada, R. Endoluminal treatment of ruptured abdominal aortic aneurysm with small intestinal submucosa sandwich endografts: a pilot study in sheep. Cardiovasc. Intervent. Radiol., 22(4), pp. 99-105, 2001.

[13] Cartes-Zumelzu, F., Lammer, J., Hoelzenbein, T., Cejna, M., Schoder, M., Thurnher, S., and Kretschmer, G. Endovascular placement of a nitinolePTFE stent-graft for abdominal aortic aneurysms: initial and midterm results. J. Vasc. Interv. Radiol., 13(5), pp. 465-473, 2002.

[14] Pétas, A., Talja, M., Tammela, T., Taari, K., Lehtoranta, K., Välimaa, T., and Törmälä, P. A randomized study to compare biodegradable selfreinforced polyglycolic acid spiral stents to suprapubic and indwelling catheters after visual laser ablation of the prostate. J. Urol., 157(1), pp. 173-176, 1997.

[15] Välimaa, T., Laaksovirta, S., Tammela, T.L., Laippala, P., Talja, M., Isotalo, T., Pétas, A., Tarri, K., and Törmälä, P. Viscoelastic memory and self-expansion of self-reinforced bioabsorbable stents. Biomater., 23(17), pp. 3575-3582, 2002.

[16] Toyota, N., Pavcnik, D., Vanalstine, W., Uchida, B., Timmermans, H., Yin, Q., Kaufman, J., Keller, F., Ito, K., and Rösch, J. Comparison of small intestinal submucosa-covered and noncovered nitinol stents in sheep iliac arteries: a pilot study. J. Vasc. Interv. Radiol., 13(5), pp. 489-498, 2002.

[17] Oka, M. Biomechanics and repair of articular cartilage. J. Orthop. Sci., 6, pp. 448-456, 2001.

[18] Roeder, R., Wolfe, J., Lianakis, N., Hinson, T., Geddes, L.A., and Obermiller, J. Compliance, elastic modulus, and burst pressure of smallintestine submucosa (SIS), small-diameter vascular grafts. J. Biomed. Mater. Res., 47(1), pp. 65-70, 1999.

[19] Lawrence-Brown, M., Semmens, J., Hartley, D, Mun, R., van Schie, G., Goodman, M., Prendergast, F., and Sieunarine, K. How is durability related to patient selection and graft design with endoluminal grafting for abdominal aortic aneurysm? Durability of Vascular and Endovascular Surgery, ed. by Greenhalgh, R., W.B. Saunders Company, Ltd., London, UK, pp. 375-385, 1999.

[20] Morris, W., Delassus, P., and McGloughlin, T. Experimental evaluation of the migration of aortic stent grafts. 2001 Advances in Bioengineering, ASME BED-50, pp. 495-496, 2001.

[21] Hadlock, T.A., Sundback, C.A., Hunter, D.A., Vacanti, J.P., and Cheney, M.L. A new artificial nerve graft containing rolled Schwann cell monolayers. Microsurgery, 21(3), pp. 96-101, 2001. 
